\title{
BMJ Open TROG 18.01 phase III randomised clinical trial of the Novel Integration of New prostate radiation schedules with adJuvant Androgen deprivation: NINJA study protocol
}

Jarad Martin, ${ }^{\oplus 1,2}$ Paul Keall, ${ }^{3}$ Shankar Siva, ${ }^{4}$ Peter Greer, ${ }^{1,5}$ David Christie, ${ }^{\odot 6}$ Kevin Moore, ${ }^{7}$ Jason Dowling, ${ }^{8}$ David Pryor, ${ }^{9}$ Peter Chong, ${ }^{10}$ Nicholas McLeod, ${ }^{10}$ Avi Raman, ${ }^{10}$ James Lynam, ${ }^{2}$ Joanne Smart, ${ }^{1}$ Christopher Oldmeadow, ${ }^{11}$ Colin I Tang, ${ }^{12}$ Declan G Murphy, ${ }^{13}$ Jeremy Millar, ${ }^{14}$ Keen Hun Tai, ${ }^{4}$ Lois Holloway, ${ }^{15}$ Penny Reeves, ${ }^{2,16}$ Amy Hayden, ${ }^{17}$ Tee Lim, ${ }^{18}$ Tanya Holt, ${ }^{19}$ Mark Sidhom ${ }^{15}$

To cite: Martin J, Keall $P$, Siva S, et al. TROG 18.01 phase III randomised clinical trial of the Novel Integration of New prostate radiation schedules with adJuvant Androgen deprivation: NINJA study protocol. BMJ Open 2019:9:e030731. doi:10.1136/ bmjopen-2019-030731

- Prepublication history for this paper is available online. To view these files please visit the journal online (http://dx.doi. org/10.1136/bmjopen-2019030731).

Received 01 April 2019 Revised 18 June 2019 Accepted 18 July 2019
D) Check for updates

(c) Author(s) (or their employer(s)) 2019. Re-use permitted under CC BY-NC. No commercial re-use. See rights and permissions. Published by BMJ.

For numbered affiliations see end of article.

\section{Correspondence to}

Professor Jarad Martin; jarad.martin@calvarymater. org.au

\section{ABSTRACT}

Introduction Stereotactic body radiotherapy (SBRT) is a non-invasive alternative to surgery for the treatment of non-metastatic prostate cancer (PC). The objectives of the Novel Integration of New prostate radiation schedules with adJuvant Androgen deprivation (NINJA) clinical trial are to compare two emerging SBRT regimens for efficacy with technical substudies focussing on MRI only planning and the use of knowledge-based planning (KBP) to assess radiotherapy plan quality.

Methods and analysis Eligible patients must have biopsy-proven unfavourable intermediate or favourable high-risk PC, have an Eastern Collaborative Oncology Group (ECOG) performance status 0-1 and provide written informed consent. All patients will receive 6 months in total of androgen deprivation therapy. Patients will be randomised to one of two SBRT regimens. The first will be $40 \mathrm{~Gy}$ in five fractions given on alternating days (SBRT monotherapy). The second will be 20 Gy in two fractions given 1 week apart followed 2 weeks later by 36 Gy in 12 fractions given five times per week (virtual high-dose rate boost (HDRB)). The primary efficacy outcome will be biochemical clinical control at 5 years. Secondary endpoints for the initial portion of NINJA look at the transition of centres towards MRI only planning and the impact of KBP on real-time (RT) plan assessment. The first 150 men will demonstrate accrual feasibility as well as addressing the KBP and MRI planning aims, prior to proceeding with total accrual to 472 patients as a phase III randomised controlled trial.

Ethics and dissemination NINJA is a multicentre cooperative clinical trial comparing two SBRT regimens for men with PC. It builds on promising results from several single-armed studies, and explores radiation dose escalation in the Virtual HDRB arm. The initial component includes novel technical elements, and will form an important platform set for a definitive phase III study.

Trial registration number ANZCTN 12615000223538.
Strengths and limitations of this study

Randomised trial comparing two emerging radiotherapy regimens for prostate cancer.

- Technological substudy seeking to implement MRI only planning.

- Use of novel approaches such as automated plan assessment to ensure high-quality treatment.

- Limitation is the use of a biochemical surrogate endpoint at 5 years rather than longer-term survival endpoints

\section{BACKGROUND}

Stereotactic body radiotherapy for prostate cancer

Prostate cancer (PC) has a major impact on the Australian population with 3500 deaths projected in 2018 and treatment costs to patients and the health system exceeding $\$ 500$ million by $2025 .{ }^{12}$ The question at the heart of Novel Integration of New prostate radiation schedules with adJuvant Androgen deprivation (NINJA) is to compare two emerging and practice-changing schedules of radiotherapy that leverage state-of-the-art technology developments and our Australian clinical trial experience to make treatments safer, highly efficient and more convenient for patients. The first schedule is a five-fraction stereotactic body radiotherapy (SBRT monotherapy) approach. ${ }^{3}$ The alternative regimen is 'virtual high-dose rate boost' (HDRB), non-invasively delivering brachytherapy-type doses. ${ }^{4}$ Superiority of the latter schedule would validate the utility of dose escalation to improve outcomes. Similarity of outcomes in 


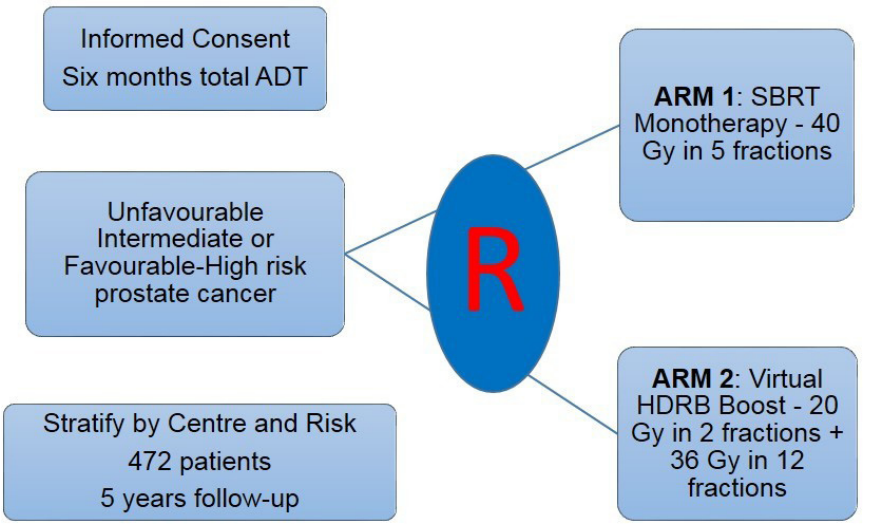

Figure 1 Novel Integration of New prostate radiation schedules with adJuvant Androgen deprivation trial schema. ADT, androgen deprivation therapy; HDRB, high-dose rate boost; SBRT, stereotactic body radiotherapy.

the former schedule would allow for major cost savings and reduced patient burden with reduction of treatment sessions from 40 to 5 (see figure 1 ).

Conventional radiotherapy regimens for $\mathrm{PC}$ are given five times per week for up to nine consecutive weeks. ${ }^{5}$ Recent results from large non-inferiority studies including substantial Australian input has helped establish a 4-week moderately hypofractionated schedule as an alternative approach. $^{6-8}$ Building on this, large series are showing excellent outcomes with regimens giving as few as five radiotherapy fractions, using higher daily doses of radiotherapy. ${ }^{910}$ A 477 patient series with median follow-up of 7 years showed $89.6 \%$ biochemical disease control with late grades 2 and 3 genitourinary (GU) toxicity low at $9 \%$ and $1.7 \%$, respectively. ${ }^{11} 12$ Grade 2 gastrointestinal (GI) toxicity was similarly favourable at $4.1 \%$. Our Stereotactic Prostate Adaptive Radiotherapy with Kilovoltage intrafraction monitoring (SPARK) phase II study used a five-fraction prostate SBRT monotherapy in conjunction with intrafraction motion management to assess the dosimetric impact of increasing the accuracy of radiotherapy dose delivery. ${ }^{3}$

Following on from this experience, several randomised studies are currently underway exploring similar stereotactic regimens, where much higher daily doses of radiotherapy are given in between five and seven visits (table 1). The Scandinavian HYPO-RT-PC study completed accrual in 2015, and presented early toxicity data in 2016 showing no significant differences between the control and SBRT arms. ${ }^{13} 14$ Initial efficacy results from this study were presented in 2018, showing no differences between the two arms. Recent guidelines have incorporated prostate SBRT monotherapy as a treatment option for centres experienced in this technique. ${ }^{15}$ Bringing this together, although SBRT monotherapy can currently be considered investigational, it is likely to gain wider acceptance as a standard treatment option in the near future. Hence, our plan is to commence NINJA as a randomised phase II study, but to convert to a fully powered phase III study with SBRT monotherapy as the control arm as the evidence base continues to mature.

Strong evidence exists for superior disease control through the use of a brachytherapy boost compared with conventional radiotherapy. ${ }^{16}$ Despite this, the use of brachytherapy continues to decline, partly due to concerns regarding higher risks of significant late GU toxicity. ${ }^{18}$ Also, the lack of evidence for improved disease control translating to improved survival has limited uptake, although the poor sensitivity of conventional staging investigations may contribute to superior local control being overwhelmed by undiagnosed micro-metastatic disease. The emergence of PSMA-PET as a more sensitive and specific staging modality makes revisiting the radiotherapy dose-escalation question highly relevant. ${ }^{1920}$ An alternative approach to brachytherapy is a 'virtual HDRB' where two to three large doses designed to mimic HDRB are delivered via stereotactic techniques with an additional fractionated external beam radiation therapy (EBRT) component. Relatively small virtual HDRB series with nearly 4-year follow-up have shown this approach to be feasible, although often using specialised equipment such as the Cyberknife platform. ${ }^{21}{ }^{22}$ Virtual HDRB has also been proven feasible in the setting of multicentre phase II trial in Australia, with 135 men enrolled on the PROMETHEUS trial (ACTRN12615000223538) where two fractions of 9.5-10 Gy are followed by an EBRT component of either 46 Gy in 23 or 36 Gy in 12 fractions. Early data from PROMETHEUS show no grades 2-3 late GI toxicity after 24 months and grade 2 late GU toxicity prevalence rates of $<7 \%$ out to 3 years. Promising efficacy signals are also becoming evident, with almost ablative prostate-specific antigen (PSA) levels being observed consistent with excellent disease response. ${ }^{23}$

Virtual HDRB may represent a significant biological dose escalation compared with SBRT monotherapy. Assuming PC has an alpha beta ratio of $1.5 \mathrm{~Gy}, 40 \mathrm{~Gy}$ in five fractions and virtual HDRB would be equivalent to 110 and 120 Gy in 2 Gy equivalent fractions, respectively. Modelling of RCT data suggests that each extra grey in

Table 1 Selected current and pending randomised trials investigating stereotactic body radiotherapy for prostate cancer

\begin{tabular}{lllll}
\hline Trial & Control arm(s) & Experimental arm & N & Progress \\
\hline HYPO-RT-PC (ISRCTN45905321) & 78 Gy/39 & 42.7 Gy/7 & 1200 & Results presented 2018 \\
PACE (NCT01584258) & 78 Gy/39 or 62 Gy/20 & 36.25 Gy/5 & 858 & Completed accrual \\
HEAT (NCT01794403) & $70.2 \mathrm{~Gy} / 26$ & $36.25 \mathrm{~Gy} / 5$ & 456 & Accruing \\
NRG GU005 (NCT03367702) & $70 \mathrm{~Gy} / 28$ & $36.25 \mathrm{~Gy} / 5$ & 622 & Accruing \\
\hline
\end{tabular}


dose translates to $\sim 2 \%$ improvement in disease control. ${ }^{24}$ The virtual HDRB approach also acknowledges the possibility of heterogeneity in the alpha-beta ratio, and therefore potentially allows for some variation in fraction size sensitivity within and between tumours. A reasonable question would be whether the excellent results seen with HDR brachytherapy boost could be safely translated into the stereotactic setting on the basis of this increase in biological dose delivery. This is the fundamental question which drives NINJA.

\section{Knowledge-based planning}

Knowledge-based planning (KBP) has the potential to simultaneously improve and automate the radiotherapy planning process. KBP uses previous cases to build a model of an optimal treatment plan which can then be applied to the current patient. Previous work suggests that KBP can provide faster and frequently better plans, ${ }^{25}$ but this has not been prospectively assessed in a multicentre fashion. NINJA provides an ideal opportunity for this.

Radiotherapy plan quality is critically important in achieving optimal treatment outcomes. The Australia-led Trans-Tasman Radiation Oncology Group (TROG) 02-02 study for patients with locally advanced head and neck cancer showed that non-protocol compliant plans had a locoregional control and overall survival decrement of $24 \%$ and $20 \%$, respectively. ${ }^{26}$ Via TROG, Australia has become leaders in the use of approaches such as stringent credentialing and RT review (RTR) of RT contours and plans, with work in PC subsequently showing very low rates of protocol deviations both in the definitive prostate and post-prostatectomy irradiation scenarios. ${ }^{27} 28$

An issue with the current RTR process is that although a plan can be deemed satisfactory, it is difficult to determine whether it could be improved. As treatment techniques evolve, satisfying the dose constraints in clinical trial protocols can become progressively easier. KBP has emerged as a promising approach to assess and improve plan quality. In KBP, a model is developed using a range of patient anatomies and target volumes. This can then be rapidly applied to a new case to either generate a plan de novo or to compare with a conventional plan. The Radiation Therapy Oncology Group (RTOG) 0126 prostate cohort was selected to study treatment plan quality variations. This work examined the high-dose Intensity Modulated Radiation Therapy (IMRT) patients using a KBP model to identify the plans that best met the dosimetric aims of the protocol. ${ }^{29}$ Focusing on Grade 2+late rectal toxicities with an outcomes-validated normal tissue complication probability model, the high-dose arm of RTOG 0126 patients treated with IMRT patients had a $15.1 \%$ cumulative incidence of Grade 2+rectal complications. ${ }^{30} \mathrm{KBP}$ plans were predicted to lead to a $4.7 \%$ risk reduction in this rate, which therefore may have cut this incidence by a third. The observed quality variations in RTOG 0126 give the strongest evidence yet that suboptimal planning is a critical problem in multi-institutional radiotherapy clinical trials and in the wider practice of radiotherapy. $\mathrm{KBP}$ has yet to be robustly assessed in a multicentre fashion, where the heterogeneity of planning systems and personnel would be expected to be greatest.

\section{MRI radiotherapy planning}

CT is widely used for radiotherapy dosimetry calculation because of the ability to directly measure electron density. Our team has validated the use of MRI to create a substitute CT (sCT) which can then be used for accurate dose calculation. ${ }^{31}$ The superior soft tissue resolution of MRI, absence of radiation dose and reduction in image artefacts means that if the dose calculation problem could be solved, standard CT-based planning would be rendered obsolete. $^{32}$

Many centres now acquire both a CT and a MRI scan for each patient, but co-registration of these datasets introduces significant error mostly under the influence of bladder filling and varying rectal distension. An attractive alternative would be to create a sCT from the MRI dataset to allow RT dose calculation. Our team has developed a hybrid atlas-voxel-based technique of sCT generation which showed high agreement in both mean monitor units $(0.3 \% \pm \mathrm{SD} 0.8 \%)$ and dose delivery (three-dimensional (3D) gamma pass rate at $2 \mathrm{~mm} / 2 \%$ level of $100 \% \pm \mathrm{SD}$ $0 \% .{ }^{31}$ A group of Swedish centres have shown similar findings in a retrospective, multicentre study, ${ }^{33}$ and our group is prospectively evaluating this approach in two centres (HIPSTER study-ACTRN12616001653459). Given the advantages of MRI for PC, and the improving access to MRI in Australia (including radiotherapy departments with dedicated planning MRI facilities), this is another area ripe for wider assessment, implementation and eventual broader application.

\section{Summary}

NINJA is a combined phase II/III multicentre study of 472 men randomised to two novel radiotherapy schedules. The hypotheses are that NINJA will advance (1) biochemical clinical control (BCC) of PC, (2) treatment planning via automation and (3) planning imaging methodology.

\section{Aim 1: radiobiological dose escalation}

The escalated radiation dose delivered using a virtual HDRB approach achieves superior disease control compared with a SBRT monotherapy alternative.

\section{Aim 2: KBP advantage}

The treatment plans using KBP will be dosimetrically superior to traditional manual planning approaches.

\section{Aim 3: MRI only planning}

MRI will give dosimetry similar to standard CT planning.

\section{METHODS/DESIGN}

\section{Study design}

The study design is a prospective randomised phase III trial which conforms to the StandardProtocol Items: 
Recommendations for Interventional Trial (SPIRIT) guidelines. We will initially enrol 150 men to demonstrate accrual feasibility as well as addressing the KBP and MRI planning aims, prior to proceeding with total accrual as a randomised phase III controlled trial.

- Stage one. Feasibility indicators- activate at least 10 centres, and accrue 50 patients within 18 months of central HREC approval.

- Stage two: Accrue total of 150 patients for randomised phase II component within 36 months of approval. Analyses of KBP and MRI planning components.

- Stage three: Complete accrual of 472 patients to the two SBRT arms.

\section{Key trial eligibility criteria}

Unfavourable intermediate or favourable high-risk PC (any combination of ISUP 3-5 and/or cT2b/T2c/ T3aN0 and/or PSA 10-20 in the absence of other highrisk factors, ie, T3b/T4, PSA >20). For high-risk patients, PSMA-PET staging prior to study entry showing NOM0 disease. Prostate volume $<100 \mathrm{cc}$, and patients can only be randomised after a plan has been generated showing that protocol compliant treatment can be performed.

\section{Pre-treatment}

All patients will receive a total of 6 months of androgen deprivation therapy (ADT). ${ }^{34} 35$ The use of PSMA-PET staging for high-risk men, and criteria to exclude very high-risk features should minimise any potential additive benefits of longer course ADT in this population. Both CT and MRI planning scans will be performed for the first 10 patients at each centre and phasing out CT for centres involved in MRI planning aspect of NINJA. Rectal displacement (eg, SpaceOAR, Rectafix, Rectal Balloon) is encouraged, but not mandated. ${ }^{36}$ Urethral positional estimation via temporary catheterisation or equivalent approaches such as high-resolution sagittal MRI can be performed. Erectile sparing RT plans for men with adequate baseline International Index of Erectile Function (IIEF) and desire to maintain erectile function can be used. ${ }^{37}$ Centres will be credentialed for MRI planning via their first five patients being primarily planned off the CT, but with sCT generation and confirmation of accurate dosimetry. The next 10 patients will have planning performed on sCT and confirmed on planning CT. Subsequent patients will omit a planning CT, be planned on sCT and have confirmation of accurate dosimetry on treatment using a centrally approved approach, for example, Electronic Portal Imaging Device (EPID) dosimetry $^{38}$ or in vivo dosimetry. ${ }^{39}$

\section{Time-dose-fractionation planning details}

Clinical target volume (CTV): Entire prostate and proximal $10 \mathrm{~mm}$ of seminal vesicles. No elective nodal irradiation permitted.

Planning target volume (PTV): For SBRT treatments, 3 mm uniform expansion from CTV. For Virtual HDRB 36
Gy in 12 fraction component, $7 \mathrm{~mm}$ uniform expansion from CTV.

SBRT Monotherapy arm: $40 \mathrm{~Gy}$ in five fractions delivered two to three times per week, prescribed to CTV D95\%.

Virtual HDRB boost arm: 20 Gy in two fractions prescribed to CTV D95\% delivered once a week followed by a 2-week break and then $36 \mathrm{~Gy}$ in 12 fractions delivered five times per week prescribed to PTV D95\%. See table 2A-C for dose constraints, and figure 2 for an example of the SBRT dosimetry.

\section{Quality assurance}

Centre credentialing will include submission of a 'Virtual HDRB' treatment plan for a patient to ensure accurate contouring and protocol compliant dose delivery. The initial KBP model will be generated from phase 2 SPARK and PROMETHEUS trials, but will be updated as NINJA proceeds. All cases will be submitted for KBP comparison, and an automated report to be returned within 24 hours. RTR will occur for all patients on trial.

\section{Treatment delivery}

All patients require intra-prostatic markers, and both inter-fraction and intra-fraction motion management strategies to ensure accurate treatment delivery. For intra-fraction motion assessment, numerous 'RT' approaches are acceptable (eg, KIM, Calypso, Cyberknife). In all instances, translational movements to be corrected to $0 \mathrm{~mm}$ threshold prior to commencing treatment. Rotational corrections do not need to be applied due to minimal dosimetric impact from such motion. ${ }^{40}$

\section{Outcome reporting}

Indicators of feasibility, accuracy, impact on replanning, and other qualitative and quantitative markers of KBP and MRI planning will be collected. Patient-reported outcomes (PROs) to include baseline and serial PROs (IIEF, Expanded Prostate Cancer Index Composite (EPIC)), physician toxicity grading (CTC AE v5), PSA and any sites of confirmed disease relapse or death due to any cause. If the prevalence of CTC AE grade 3 GI or GU toxicities exceeds $10 \%$ at any stage, the trial will be halted for safety assessment. A SPIRIT flowchart is presented in table 3 .

\section{Statistical considerations}

The statistical justification required to achieve the primary efficacy endpoint (aim 1) is as follows. BCC is a hybrid of biochemical failure via the nadir plus two definition, deployment of salvage treatments, or the detection of local, regional or metastatic relapse via imaging. Using a similar endpoint as well as a short course of ADT, the CHHiP study 60 Gy arm had $90.2 \%$ and $84.2 \%$ BCC for intermediate-risk and high-risk patients, respectively. ${ }^{41}$ The Androgen Suppression Combined with Elective Nodal and Dose Escalated Radiation Therapy study also included intermediate-risk and high-risk men, managed with 12 months of ADT, and the experimental arm delivered $46 \mathrm{~Gy}$ in 23 fractions of EBRT alongside a 
Table 2A Target volume objectives and Organs At Risk (OAR) constraints-40 Gy in 5\#

\begin{tabular}{|c|c|c|c|}
\hline Objective & Protocol & Minor variation & Major variation \\
\hline CTVp D95\% & $\geq 40.0$ & $38 \leq 40$ Gy & $<38$ Gy \\
\hline PTV_4000 D95\% & $\geq 36$ Gy & $34.44 \leq 36$ Gy & $<34.44$ Gy \\
\hline PTV_4000 D98\% & $\begin{array}{l}\geq 34.44 \text { Gy } \\
\text { (95\% of } 36.25 \text { Gy) }\end{array}$ & $32.72 \leq 34.44 \mathrm{~Gy}$ & $<32.72$ Gy \\
\hline PTV_4000 D2\% & $\leq 42 \mathrm{~Gy}$ & 42-42.8 Gy & $>42.8 \mathrm{~Gy}$ \\
\hline $\operatorname{Dmax}(0.1 \mathrm{cc})$ & $\leq 42.8 \mathrm{~Gy}$ & 42.8-44 Gy & $>44$ Gy \\
\hline $\operatorname{Dmax}(0.1 \mathrm{cc})$ & Not in OAR & NA & In OAR \\
\hline Constraint & Protocol & Minor variation & Major variation \\
\hline RECTUM V40 Gy & $\leq 0.1 \mathrm{cc}$ & NA & $>0.1 \mathrm{cc}$ \\
\hline RECTUM V36 Gy & $\leq 1 \mathrm{cc}$ & $>1-2 c c$ & $>2 \mathrm{cc}$ \\
\hline RECTUM V32 Gy & $\leq 10 \%$ & $>10 \%-20 \%$ & $>20 \%$ \\
\hline RECTUM V20 Gy & $\leq 40 \%$ & $>40 \%-50 \%$ & $>50 \%$ \\
\hline URETHRA_PRV V42 Gy & $\leq 0.1 \mathrm{cc}$ & NA & $>0.1 \mathrm{cc}$ \\
\hline BLADDER V40 Gy & $\leq 2 \mathrm{cc}$ & $>2-3 c c$ & $>3 c c$ \\
\hline BLADDER V36 Gy & $\leq 10 \mathrm{cc}$ & $>10-20 c c$ & $>20 c c$ \\
\hline BLADDER V32 Gy & $\leq 10 \%$ & $>5 \%-10 \%$ & $>10 \%$ \\
\hline BLADDER V20 Gy & $\leq 40 \%$ & $>40 \%-50 \%$ & $>50 \%$ \\
\hline PENILE BULB V36 Gy & $\leq 0.1 \mathrm{cc}$ & NA & $>0.1 \mathrm{cc}$ \\
\hline PENILE BULB V20 Gy & $\leq 3 c c$ & $>3-5 c c$ & $>5 \mathrm{cc}$ \\
\hline FEM HEAD V30 Gy & $\leq 0.1 \mathrm{cc}$ & NA & $>0.1 \mathrm{cc}$ \\
\hline FEM HEAD V20 Gy & $\leq 10 \mathrm{cc}$ & $>10-15 c c$ & $>15 \mathrm{cc}$ \\
\hline SIGMOID V40 Gy & $\leq 0.1 \mathrm{cc}$ & NA & $>0.1 \mathrm{cc}$ \\
\hline SIGMOID V36 Gy & $\leq 2 \mathrm{cc}$ & $>2-3 c c$ & $>3 c c$ \\
\hline \multicolumn{4}{|l|}{ Constraint } \\
\hline SMALL BOWEL V30 Gy & $\leq 1 \mathrm{cc}$ & NA & $>1 c c$ \\
\hline SMALL BOWEL V25 Gy & $\leq 20 \mathrm{cc}$ & $>20-40 \mathrm{cc}$ & $>40 \mathrm{cc}$ \\
\hline Conformity index ${ }^{\star}$ & $\leq 1.1$ & $>1.1-1.2$ & $>1.2$ \\
\hline Int. dose spillage† & $\leq 4$ & $>4-5$ & $>5$ \\
\hline MU/cGy ratioł & $\leq 3$ & $>3-4$ & $>4$ \\
\hline
\end{tabular}

*Optional-volume receiving 36.25 Gy/volume of PTV.

†Optional-ratio of volume receiving 36.25 Gy: 18.13 Gy.

$\ddagger$ Optional-ratio of Monitor Units (MU) delivered per fraction divided by 800 (the number of centiGray (cGy) prescribed/fraction).

CTV, clinical target volume; PTV, planning target volume.

Table 2B Target volume objectives and organs at risk constraints - virtual high-dose rate boost, stereotactic body radiotherapy component 20 Gy in two fractions

\begin{tabular}{llll}
\hline Objective & Protocol & Minor variation & Major variation \\
\hline CTVp D95\% & $\geq 20 \mathrm{~Gy}$ & $18 \leq 20 \mathrm{~Gy}$ & $<18 \mathrm{~Gy}$ \\
PTV_2000 D95\% & $\geq 18 \mathrm{~Gy}$ & $17 \leq 18 \mathrm{~Gy}$ & $<17 \mathrm{~Gy}$ \\
\hline PTV_2000 D98\% & $\geq 17 \mathrm{~Gy}$ & $16 \leq 17 \mathrm{~Gy}$ & $<16 \mathrm{~Gy}$ \\
PTV_2000 D2\% & $\leq 21 \mathrm{~Gy}$ & $>21-21.4 \mathrm{~Gy}$ & $>21.4 \mathrm{~Gy}$ \\
Dmax (0.1cc) & $\leq 21.4 \mathrm{~Gy}$ & $>21.4-22 \mathrm{~Gy}$ & $>22 \mathrm{~Gy}$ \\
Dmax (0.1cc) & Not in OAR & NA & In OAR \\
Constraint & & & $>0.1 \mathrm{cc}$ \\
RECTUM V20 Gy & $\leq 0.1 \mathrm{cc}$ & NA & $>2 \mathrm{cc}$ \\
RECTUM V16 Gy & $\leq 1 \mathrm{cc}$ & $>1-2 \mathrm{cc}$ & $>20 \%$ \\
RECTUM V14 Gy & $\leq 10 \%$ & $>10 \%-20 \%$ & $\mathrm{C}$
\end{tabular}


Open access

\begin{tabular}{llll}
\hline Table 2B Continued & & & \\
\hline Objective & Protocol & Minor variation & Major variation \\
\hline RECTUM V10 Gy & $\leq 40 \%$ & $>40 \%-50 \%$ & $>50 \%$ \\
URETHRA_PRV V21 Gy & $\leq 0.1 \mathrm{cc}$ & $\mathrm{NA}$ & $>0.1 \mathrm{cc}$ \\
BLADDER V20 Gy & $\leq 2 \mathrm{cc}$ & $>2-3 \mathrm{cc}$ & $>3 \mathrm{cc}$ \\
\hline BLADDER V18 Gy & $\leq 10 \mathrm{cc}$ & $>10-20 \mathrm{cc}$ & $>20 \mathrm{cc}$ \\
BLADDER V16 Gy & $\leq 10 \%$ & $>10 \%-15 \%$ & $>15 \%$ \\
BLADDER V10 Gy & $\leq 40 \%$ & $>40 \%-50 \%$ & $>50 \%$ \\
PENILE BULB V18 Gy & $\leq 0.1 \mathrm{cc}$ & $\mathrm{NA}$ & $>0.1 \mathrm{cc}$ \\
PENILE BULB V10 Gy & $\leq 3 \mathrm{cc}$ & $>3-5 \mathrm{cc}$ & $>5 \mathrm{cc}$ \\
FEM HEAD V15 Gy & $\leq 0.1 \mathrm{cc}$ & $\mathrm{NA}$ & $>0.1 \mathrm{cc}$ \\
FEM HEAD V10 Gy & $\leq 10 \mathrm{cc}$ & $>10-15 \mathrm{cc}$ & $>15 \mathrm{cc}$ \\
SIGMOID V20 Gy & $\leq 0.1 \mathrm{cc}$ & $\mathrm{NA}$ & $>0.1 \mathrm{cc}$ \\
SIGMOID V18 Gy & $\leq 2 \mathrm{cc}$ & $>2-3 \mathrm{cc}$ & $>3 \mathrm{cc}$ \\
SMALL BOWEL V15 Gy & $\leq 1 \mathrm{cc}$ & $\mathrm{NA}$ & $>1 \mathrm{cc}$ \\
SMALL BOWEL V10 Gy & $\leq 20 \mathrm{cc}$ & $>20-40 \mathrm{cc}$ & $>40 \mathrm{cc}$ \\
Conformity index & $\leq 1.1$ & $>1.1-1.2$ & $>1.2$ \\
Int. dose spillage† & $\leq 4$ & $>4-5$ & $>5$ \\
\hline MU/cGy ratio & $\leq 3$ & $>3-4$ & $>4$ \\
\hline
\end{tabular}

*Optional-volume receiving 18 Gy/volume of PTV.

†Optional-ratio of volume receiving $18 \mathrm{~Gy}: 9$ Gy.

$\ddagger$ Optional-ratio of MU delivered per fraction divided by 1000 (the number of cGy prescribed/fraction).

CTV, cilinical target volume; PTV, planning target volume.

Table 2C Target volume objectives and organs at risk constraints - virtual HDRB, EBRT component 36 Gy in 12 fractions

\begin{tabular}{llll} 
Objectives & Protocol & Minor variation & Major variation \\
\hline PTV_3600 D95\% & $\geq 36 \mathrm{~Gy}$ & $34.2 \leq 36 \mathrm{~Gy}$ & $<34.2 \mathrm{~Gy}$ \\
PTV_3600 D98\% & $\geq 34.2 \mathrm{~Gy}$ & $32.4 \leq 34.2 \mathrm{~Gy}$ & $<32.4 \mathrm{~Gy}$ \\
PTV_3600 D2\% & $\leq 37.8 \mathrm{~Gy}$ & $>37.8-38.5 \mathrm{~Gy}$ & $>38.5 \mathrm{~Gy}$ \\
PTV_3600 $(0.1 \mathrm{cc})$ & $\leq 38.5 \mathrm{~Gy}$ & $>38.5-39.6 \mathrm{~Gy}$ & $>39.6 \mathrm{~Gy}$ \\
Constraint & & & \\
Small Bowel Dmax $(0.1 \mathrm{cc})$ & $\leq 36 \mathrm{~Gy}$ & $>36-38 \mathrm{~Gy}$ & $>38 \mathrm{~Gy}$ \\
Fem Head Dmax $(0.1 \mathrm{cc})$ & $\leq 25 \mathrm{~Gy}$ & $>25-35 \mathrm{~Gy}$ & $>35 \mathrm{~Gy}$ \\
Rectum V30 Gy & $\leq 25 \%$ & $>25 \%-35 \%$ & $>35 \%$ \\
Bladder V32 Gy & $\leq 25 \%$ & $>25 \%-35 \%$ & $>35 \%$ \\
\hline
\end{tabular}

CTV, cilical target volume; PTV, planning target volume.

LDR brachytherapy boost. ${ }^{17}$ At 5 years, the BCC was $89 \%$ in the brachytherapy boost cohort, although with a higher-risk patient mix than we are going to accrue on this protocol. Allowing for differences in inter-trial comparisons, we estimate BCC $\sim 86 \%$ in the standard SBRT arm. Similar data have been reported for single-arm SBRT monotherapy series. ${ }^{12}$ For a superiority RCT design, we will aim for a HR of 0.5 in 5-year BCC for the virtual HDR arm that is, $93 \%$. An HR of 0.5 is chosen because this translates to an absolute improvement of $7 \%$, and any improvements less than this are unlikely to be clinically significant. With alpha 0.05 , power of $80 \%$, and dropout of $2 \%$ the required phase III sample size is 472 men.
For KBP (aim 2), we hypothesise that a replanning rate of $>15 \%$ would be clinically significant. Assuming an error rate of $\pm 6 \%$, at an alpha of $5 \%, 136$ patients are required. Allow $10 \%$ dropout due to technical issues with a new planning paradigm: total of 150 cases. For MRI planning (aim 3), having $\geq 50 \%$ of centres involved in this aspect of NINJA completely transition to MRI only planning will be deemed a success.

Endpoints NINJA aim 1: radiobiological dose escalation For each patient visit, PSA, GU and GI RTOG physician graded toxicity and PROs using the EPIC instrument will be recorded. The acute toxicity will be measured each 


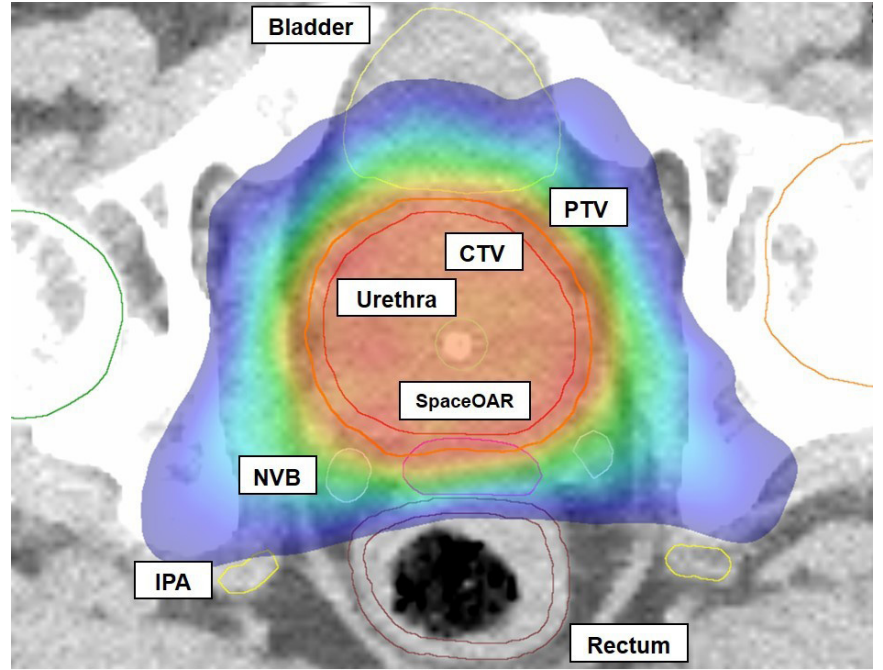

Figure 2 Novel Integration of New prostate radiation schedules with adJuvant Androgen deprivation dosimetry example showing very conformal nature of high-dose treatment to the PTV. CTV, clinical target volume; IPA, internal pudendal artery; NVB, neurovascular bundle; PTV, planning target volume.

week of treatment, and 2 weeks after treatment completion. As severe acute toxicity is a surrogate for late toxicity, this will be the primary physician-reported toxicity outcome for this 3-year study. PROs will be at baseline, then 1-year, 3-year and 5-year marks. Biochemical control will be assessed with PSA testing at baseline, then every 6 months, with failure defined by the nadir plus 2 Phoenix definition. Clinical control consists of any evidence of relapse on imaging, or the initiation of salvage treatments. BCC is the combination of either biochemical or clinical events. BCC at 5 years will be the primary endpoint for aim 1 .

\section{Endpoint NINJA aim 2: KBP advantage}

KBP models will initially be developed for the SBRT monotherapy and virtual HDRB arms. The training sample for the NINJA KBP model will come from the SPARK and PROMETHEUS cohorts, and will be continuously improved during the NINJA trial. As new cases are accepted to the trial, they will be incorporated into the knowledge-based dose prediction models to broaden the geometric experience and improve future prediction accuracy. The NINJA KBP automated planning routines' performance will be validated on an independent validation sample of cases (holding back 20\% of SPARK/ PROMETHEUS cases) to ensure that the final KBP plans are effecting plans that match the dosimetric goals of the NINJA protocol.

All NINJA patients will have a plan generated as per local standard of care by the treating centre. If sites are capable of utilising KBP locally, they will be provided with the NINJA KBP routine. All plans will then be uploaded to TROG to be compared with a KBP-generated plan. If the site was submitting a manually generated plan, an automated report will be returned to the treating centre within 24 hours, at which time they can decide whether to proceed with their original manual plan or to replan based on the KBP recommendations. If the site utilises the NINJA KBP routine, a central quality check will be performed to ensure proper use of the model, but no further recommendation will be made to the submitting site. The utility of KBP will be assessed by recording the rate of replanning following receipt of the KBP plan.

\section{Endpoint NINJA aim 3: - MRI planning validation}

This substudy will be for centres with access to MRI scanning with appropriate accessories such as a flat top couch. Patients will have a CT and a MRI performed in

Table 3 Schedule of assessments as per StandardProtocol Items: Recommendations for Interventional Trial guidelines

Pre-treatment Follow-up

24, 60

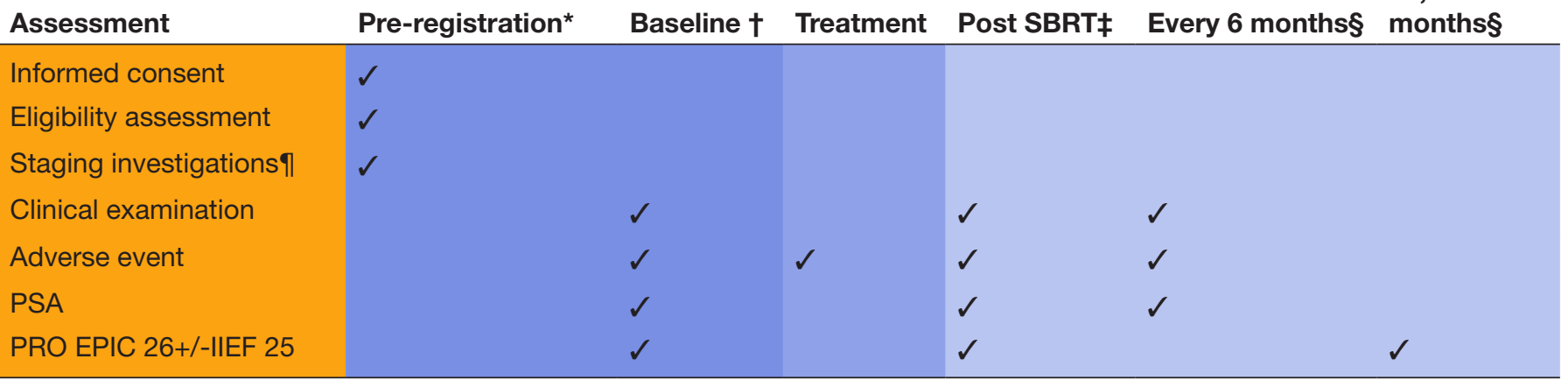

${ }^{*}$ To be done within 60 days of registration.

†To be done no more than 2 weeks post-registration and within 4 weeks of starting treatment.

$\ddagger$ To be performed between 6 weeks post-SBRT treatment completion.

§From commencement of androgen deprivation therapy.

INote that PSMA-PET is mandated for high-risk patients. Whole body bone scan with CT or MRI of the pelvis \pm abdomen are acceptable for unfavourable intermediate risk patients.

EPIC, Expanded Prostate Cancer Index Composite; PSA, prostate-specific antigen; SBRT, stereotactic body radiotherapy. 
the planning position. Clinicians will contour all target volumes and organs at risk on the MRI. Sites who have not been validated for MRI-based planning will go through a credentialing phase, where the first five patients will have the planning processes assessed. ${ }^{31}$ Following credentialing (or evidence of previously fulfilling this requirement), the MRI will be exported for remote generation of a sCT. A plan will then be created on the sCT, and copied onto the planning CT. The dosimetry of these will be compared at points within both the target volume and critical structures. If the isocentre dose is within $2 \%$ and $3 \mathrm{D}$ gamma comparison at $2 \% / 2 \mathrm{~mm}$ criteria $>90 \%$ passrate for the entire scanned volume, then the sCT plan will be deemed accurate and used for patient treatment. After 10 such patients, centres will have the option of no longer performing a routine planning scan, but instead using in vivo dosimetry to confirm accurate dose delivery with the same criteria as for the sCT and planning CT comparison. The utility of MRI planning will be assessed via:

- Accuracy-The proportion of plans where both the isocentre dose and gamma comparison are within the stated constraints. Deemed accurate if $>95 \%$.

- Feasibility-The proportion of sites who commence accrual who subsequently (a) achieve credentialing and (b) move successfully completely to MR only planning. Deemed feasible is $\geq 50 \%$ of sites.

\section{Other substudies}

- PROs using the IIEF and EPIC questionnaire.

- Physician-reported toxicity using the CTC AE v5 scale.

- Health economics-the cost-effectiveness profiles of the technologies being compared will be assessed in a cost consequence analysis. Resource use implications and impacts have utility both for decision-makers and for informing the phase III trial-based economic evaluation.

- Erectile sparing RT (neurovascular bundles, pudendal arteries, penile bulb) and impact on PROs

- Performance comparison between intrafraction motion management strategies

\section{Patient and public involvement}

Many of the baseline requirements for NINJA have been informed by consumer feedback. The concept of improved treatment accuracy resonated with our consumer advisors, and as such is mandated for all patients in NINJA. Improved pre-treatment imaging with PSMA-PET will help define those most likely to benefit from aggressive management of their primary PC, an approach which our consumer advisors found essential for men with higher-risk disease. Our consumer advisors also prioritise PROs, and as such, PROs are one of our key endpoints. Our focus on assessing shorter, non-invasive radiotherapy treatment regimens which can be delivered on an outpatient basis also resonated with our consumer advisors.

Our consumer advisors will engage with consumer groups through organisations such as TROG, Australia
New Zealand Urogenital Programme and Prostate Cancer Foundation of Australia to ensure broad consumer awareness of NINJA. The Trial Management Committee will continue to include our consumer advisors in ongoing discussions regarding accrual and toxicities to gain their perspective on any changes to the conduct of the trial which might be advisable.

NINJA is designed with numerous potentially practice changing outcomes; consumers will remain critical throughout the trial to maximise integration of these into wider clinical practice. Several of our team are very active on social media, which can make direct connexions with consumers about our findings. Many of our clinician CIs and AIs are regular speakers for local PG support groups.

\section{DISCUSSION}

Prostate SBRT, KBP and MRI planning are all highly promising approaches with the potential to transform patient care far beyond the specific indication of definitive PC management. A large array of substudies will create new scientific knowledge and further inform best practice PC radiotherapy. The study plan seeks to assess and validate all of these approaches. More importantly, we aim to increase the capabilities of centres to perform such leading edge treatments. If validated, these approaches can be seamlessly integrated into routine clinical practice.

Conventional PC radiotherapy currently takes between 20 and 40 outpatient visits, so reducing this to between 5 and 14 will assist with access for patients as well as improve resource utilisation. Semiautomation of the planning process via KBP will both streamline processes and reduce variable plan quality. NINJA has been deliberately designed to facilitate treatment at small and larger institutes, crossing the divide between public and private as well as metropolitan and regional.

NINJA seeks to prospectively assess and validate promising new technologies as part of a randomised study comparing two novel prostate RT regimens. The research pathway established can serve as a template for future attempts to explore promising technological innovations in a cost-effective manner. Beyond the geographical sector and regional collaborations, NINJA brings together multiple states, as well as disciplines in clinical, technical and research fields.

\section{Author affiliations}

${ }^{1}$ Department of Radiation Oncology, Calvary Mater Newcastle, Newcastle, New South Wales, Australia

${ }^{2}$ School of Medicine and Public Health, University of Newcastle, Callaghan, New South Wales, Australia

${ }^{3}$ Radiation Physics Laboratory, University of Sydney, Sydney, New South Wales, Australia

${ }^{4}$ Sir Peter MacCallum Department of Oncology, University of Melbourne, Melbourne, Victoria, Australia

${ }^{5}$ School of Mathematical and Physical Sciences, University of Newcastle, Callaghan, New South Wales, Australia

${ }^{6}$ Genesis Care, Southport, Queensland, Australia

${ }^{7}$ Department of Medical Physics, University of California San Diego, La Jolla, California, USA 
${ }^{8}$ The Australian e-Health Research Centre, CSIR0, Canberra, Australian Capital Territory, Australia

${ }^{9}$ Department of Radiation Oncology, Princess Alexandra Hospital Health Service District, Woolloongabba, Queensland, Australia

${ }^{10}$ Department of Urology, John Hunter Hospital, New Lambton Heights, New South Wales, Australia

${ }^{11}$ Hunter Medical Research Institute, Newcastle, New South Wales, Australia

${ }^{12}$ Department of Radiation Oncology, Sir Charles Gairdner Hospital, Perth, Western Australia, Australia

${ }^{13}$ Urological Service Team, Peter MacCallum Cancer Centre, Melbourne, Victoria, Australia

${ }^{14}$ Department of Radiation Oncology, Alfred Health, Melbourne, Victoria, Australia

${ }^{15}$ Department of Radiation Oncology, Liverpool Hospital, Liverpool, New South

Wales, Australia

${ }^{16}$ Department of Health Research Economics, University of Newcastle Hunter Medical Research Institute, New Lambton, New South Wales, Australia

${ }^{17}$ Department of Radiation Oncology, Westmead Hospital, Westmead, New South Wales, Australia

${ }^{18}$ Genesis Care, Perth, Western Australia, Australia

${ }^{19}$ Radiation Oncology Princess Alexandra Raymond Terrace, Brisbane, Queensland, Australia

Acknowledgements The study sponsor is the Trans-Tasman Radiation Oncology Group (TROG). The full protocol is available through TROG. We acknowledge the contributions of our consumer advisors Steve McCluskey and Russell Flank for their contributions to this clinical trial design.

Contributors JM and MS conceived of the study. JM, MS, JS and DP were responsible for protocol drafting and data management plan. JM, PK, SS, PG, DC, KM, JD, DP, PC, NM, AR, JL, JS, CO, CIT, DGM, JM, KHT, LH, PR, AH, TL, TH and MS were involved in the study design, acquisition of competitive grant funding and helped to draft this manuscript. JM, PK, SS, PG, DC, KM, JD, DP, PC, NM, AR, JL, JS, CO, CIT, DGM, JM, KHT, LH, PR, AH, TL, TH and MS will remain involved in the conduct and reporting of the study, and have read and approved the final manuscript.

Funding This study underwent competitive peer review by the National Health and Medical Research Council (NHMRC) and was awarded governmental Cancer Australia funding (APP1158455). Additional seed funding was provided by mundipharma.

\section{Competing interests None declared.}

Patient consent for publication Not required.

Ethics approval This study has received Ethical approval from the South West Sydney Local Health District Human Research Ethics Committee (approval number HREC/18/LP0OL/420). All patients will sign a Participant Information Consent Form prior to being randomised and treated on this study.

Provenance and peer review Not commissioned; externally peer reviewed.

Open access This is an open access article distributed in accordance with the Creative Commons Attribution Non Commercial (CC BY-NC 4.0) license, which permits others to distribute, remix, adapt, build upon this work non-commercially, and license their derivative works on different terms, provided the original work is properly cited, appropriate credit is given, any changes made indicated, and the use is non-commercial. See: http://creativecommons.org/licenses/by-nc/4.0/.

\section{REFERENCES}

1. Australia C. Prostate cancer statistics: prostate cancer in Australia. Available: https://prostate-cancer.canceraustralia.gov.au/statistics [Accessed 24 Feb 2018].

2. Economics $\mathrm{CfAH}$. Economic modelling of healthcare services for prostate cancer: prostate cancer Foundation of Australia, 2016.

3. Keall P, Nguyen DT, O'Brien R, et al. Stereotactic prostate adaptive radiotherapy utilising kilovoltage intrafraction monitoring: the TROG 15.01 spark trial. BMC Cancer 2017;17:180.

4. Richardson M, Sidhom M, Gallagher S, et al. Prostate multicentre external beam radioTHErapy using a stereotactic boost: the prometheus study protocol. BMC Cancer 2018;18:588.

5. Martin JM, Bayley A, Bristow R, et al. Image guided dose escalated prostate radiotherapy: still room to improve. Radiat Oncol 2009;4.

6. Catton CN, Lukka H, Gu C-S, et al. Randomized trial of a hypofractionated radiation regimen for the treatment of localized prostate cancer. Journal of Clinical Oncology 2017;35:1884-90.
7. Martin JM, Supiot S, Keall PJ, et al. Moderately hypofractionated prostate external-beam radiotherapy: an emerging standard. $\mathrm{Br} J$ Radiol 2018;58:20170807.

8. Pryor DI, Turner SL, Tai KH, et al. Moderate hypofractionation for prostate cancer: a user's guide. J Med Imaging Radiat Oncol 2018;62:232-9.

9. King CR, Collins S, Fuller D, et al. Health-Related quality of life after stereotactic body radiation therapy for localized prostate cancer: results from a multi-institutional Consortium of prospective trials. Int J Radiat Oncol Biol Phys 2013;87:939-45.

10. King CR, Freeman D, Kaplan I, et al. Stereotactic body radiotherapy for localized prostate cancer: pooled analysis from a multiinstitutional Consortium of prospective phase II trials. Radiotherapy and Oncology 2013;109:217-21.

11. Katz AJ, Kang J. Quality of life and toxicity after SBRT for organconfined prostate cancer, a 7-year study. Front Oncol 2014;4:301.

12. Katz AJ, Kang J. Stereotactic body radiotherapy as treatment for organ confined low- and intermediate-risk prostate carcinoma, a 7-year study. Front Oncol 2014;4:240.

13. Widmark A, Gunnlaugsson A, Beckman L, et al. Extreme Hypofractionation versus Conventionally Fractionated Radiotherapy for Intermediate Risk Prostate Cancer: Early Toxicity Results from the Scandinavian Randomized Phase III Trial "HYPO-RT-PC". Int J Radiat Oncol Biol Phys 2016;96:938-9.

14. Widmark A, Gunnlaugsson A, Beckman L, et al. OC-0599: Ultrahypofractionation for prostate cancer: outcome from the Scandinavian phase 3 HYPO-RT-PC trial. Radiotherapy and Oncology 2018;127.

15. Morgan SC, Hoffman K, Loblaw DA, et al. Hypofractionated radiation therapy for localized prostate cancer: Executive summary of an ASTRO, ASCO, and AUA evidence-based guideline. Pract Radiat Oncol 2018;8:354-60.

16. Spratt DE, Zumsteg ZS, Ghadjar P, et al. Comparison of highdose (86.4 Gy) IMRT vs combined brachytherapy plus IMRT for intermediate-risk prostate cancer. BJU Int 2014;13:n/a-7.

17. Morris WJ, Tyldesley S, Rodda S, et al. Androgen Suppression Combined with Elective Nodal and Dose Escalated Radiation Therapy (the ASCENDE-RT Trial): An Analysis of Survival Endpoints for a Randomized Trial Comparing a Low-Dose-Rate Brachytherapy Boost to a Dose-Escalated External Beam Boost for High- and Intermediate-risk Prostate Cancer. Int J Radiat Oncol Biol Phys 2017;98:275-85

18. Rodda S, Tyldesley S, Morris WJ, et al. ASCENDE-RT: an analysis of treatment-related morbidity for a randomized trial comparing a lowdose-rate brachytherapy boost with a dose-escalated external beam boost for high- and intermediate-risk prostate cancer. Int J Radiat Oncol Biol Phys 2017;98:286-95.

19. Perera M, Papa N, Christidis D, et al. Sensitivity, Specificity, and Predictors of Positive ${ }^{68} \mathrm{Ga}$-Prostate-specific Membrane Antigen Positron Emission Tomography in Advanced Prostate Cancer: A Systematic Review and Meta-analysis. Eur Urol 2016;70:926-37.

20. Gupta SK, Watson T, Denham J, et al. Prostate-Specific membrane antigen positron emission tomography-computed tomography for prostate cancer: distribution of disease and implications for radiation therapy planning. Int J Radiat Oncol Biol Phys 2017;99:701-9.

21. Pasquier D, Nickers $P$, Peiffert $D$, et al. Hypofractionated stereotactic boost in intermediate risk prostate carcinoma: preliminary results of a multicenter phase II trial (CKNO-PRO). PLoS One 2017;12:e0187794.

22. Paydar I, Pepin A, Cyr RA, et al. Intensity-Modulated radiation therapy with stereotactic body radiation therapy boost for unfavorable prostate cancer: a report on 3-year toxicity. Front Oncol 2017;7:5.

23. Pryor D, Sidhom M, Arumugam S, et al. Phase 2 multicenter study of Gantry-Based stereotactic radiotherapy boost for intermediate and high risk prostate cancer (prometheus). Front Oncol 2019;9:217.

24. Trada Y, Plank A, Martin J. Defining a dose-response relationship for prostate external beam radiotherapy. J Med Imaging Radiat Oncol 2013;57:237-46.

25. Li N, Carmona R, Sirak I, et al. Highly efficient training, refinement, and validation of a knowledge-based planning quality-control system for radiation therapy clinical trials. Int J Radiat Oncol Biol Phys 2017:97:164-72

26. Peters LJ, O'Sullivan B, Giralt J, et al. Critical impact of radiotherapy protocol compliance and quality in the treatment of advanced head and neck cancer: results from TROG 02.02. Journal of Clinical Oncology 2010;28:2996-3001.

27. Martin J, Frantzis J, Chung P, et al. Prostate radiotherapy clinical trial quality assurance: how real should real time review be? (a TROG-OCOG intergroup project). Radiotherapy and Oncology 2013;107:333-8. 
28. Trada $\mathrm{Y}$, Kneebone A, Paneghel A, et al. Optimizing radiation therapy quality assurance in clinical trials: a TROG 08.03 RAVES substudy. Int J Radiat Oncol Biol Phys 2015;93:1045-51.

29. Moore KL, Schmidt R, Moiseenko V, et al. Quantifying unnecessary normal tissue complication risks due to suboptimal planning: a secondary study of RTOG 0126. Int J Radiat Oncol Biol Phys 2015;92:228-35.

30. Michalski JM, Yan Y, Watkins-Bruner D, et al. Preliminary toxicity analysis of 3-dimensional conformal radiation therapy versus intensity modulated radiation therapy on the high-dose arm of the radiation therapy Oncology Group 0126 prostate cancer trial. Int J Radiat Oncol Biol Phys 2013;87:932-8.

31. Dowling JA, Sun J, Pichler P, et al. Automatic substitute computed tomography generation and contouring for magnetic resonance imaging (MRI)-Alone external beam radiation therapy from standard MRI sequences. Int J Radiat Oncol Biol Phys 2015;93:1144-53.

32. Khoo ELH, Schick K, Plank AW, et al. Prostate contouring variation: can it be fixed? Int J Radiat Oncol Biol Phys 2012;82:1923-9.

33. Persson E, Gustafsson C, Nordström F, et al. MR-OPERA: a Multicenter/Multivendor validation of magnetic resonance ImagingOnly prostate treatment planning using synthetic computed tomography images. Int J Radiat Oncol Biol Phys 2017;99:692-700.

34. Bolla M, Maingon P, Carrie C, et al. Short androgen suppression and radiation dose escalation for intermediate- and high-risk localized prostate cancer: results of EORTC trial 22991. J Clin Oncol 2016;34:1748-56.
35. Denham JW, Joseph D, Lamb DS, et al. Short-Term androgen suppression and radiotherapy versus intermediate-term androgen suppression and radiotherapy, with or without zoledronic acid, in men with locally advanced prostate cancer (TROG 03.04 radar): 10year results from a randomised, phase 3, factorial trial. Lancet Oncol 2019;20:267-81.

36. Wilton L, Richardson M, Keats S, et al. Rectal protection in prostate stereotactic radiotherapy: a retrospective exploratory analysis of two rectal displacement devices. J Med Radiat Sci 2017;64:266-73.

37. Spratt DE, Lee JY, Dess RT, et al. Vessel-sparing radiotherapy for localized prostate cancer to preserve erectile function: a single-arm phase 2 trial. Eur Urol 2017;72:617-24.

38. Woodruff HC, Fuangrod T, Van Uytven E, et al. First experience with real-time EPID-Based delivery verification during IMRT and VMAT sessions. International Journal of radiation oncology, biology. Physics 2015;93:516-22.

39. Legge K, Greer PB, O'Connor DJ, et al. Real-Time in vivo rectal wall dosimetry using MOSkin detectors during linac based stereotactic radiotherapy with rectal displacement. Radiat Oncol 2017;12.

40. Wolf $\mathrm{J}$, Nicholls $\mathrm{J}$, Hunter P, et al. Dosimetric impact of intrafraction rotations in stereotactic prostate radiotherapy: a subset analysis of the TROG 15.01 spark trial. Radiother Oncol 2019;136:143-7.

41. Dearnaley D, Syndikus I, Mossop H, et al. Conventional versus hypofractionated high-dose intensity-modulated radiotherapy for prostate cancer: 5-year outcomes of the randomised, non-inferiority, phase 3 CHHiP trial. Lancet Oncol 2016;17:1047-60. 